\title{
SSR1 Cryomodule Design for PXIE
}

\author{
Thomas H. Nicol \\ Fermi National Accelerator Laboratory \\ P.O. Box 500 \\ Batavia, IL 60510 \\ USA
}

\section{INTRODUCTION}

Fermilab is in the process of designing a Project X Injector Experiment (PXIE), a CW linac, to validate the Project $\mathrm{X}$ concept, reduce technical risks, and obtain experience in the design and operation of a superconducting proton linac. The overall facility will include an ion source, low and medium-energy beam transport sections, an RF quadrupole, and two superconducting cavity cryomodules. One will contain eight half-wave resonators operating at $162.5 \mathrm{MHz}$ and eight superconducting solenoids. The second will contain eight single spoke resonators (SSR1) operating at $325 \mathrm{MHz}$ and four superconducting solenoids. The design of the cryomodule being developed to house the $325 \mathrm{MHz}$ single spoke resonators and all related systems and services is described here.

The SSR1 cryomodule will operate with continuous wave (CW) RF power and support peak currents of $5 \mathrm{~mA}$ chopped with arbitrary patterns to yield an average beam current of $1 \mathrm{~mA}$. The RF coupler design employed should support a future upgrade path with average currents as high as $5 \mathrm{~mA}$. The RF power per cavity at 1 $\mathrm{mA}$ average current and 2.2 MV accelerating voltage $(\beta=0.22)$ should not exceed 4 KW with an overhead reserved for microphonics control. The RMS normalized bunch emittance at the $\mathrm{CM}$ exit should not exceed $0.25 \mathrm{~mm}$ mrad for each of 3 planes.

The current beam optics design for Project X requires that the SSR1 cryomodule contains eight cavities $(\mathrm{C})$ and four solenoids $(\mathrm{S})$ in the following order: $\mathrm{C}-\mathrm{S}-\mathrm{C}-$ $\mathrm{C}-\mathrm{S}-\mathrm{C}-\mathrm{C}-\mathrm{S}-\mathrm{C}-\mathrm{C}-\mathrm{S}-\mathrm{C}$. Horizontal and vertical dipole corrector are located inside each solenoid. A four-electrode beam position monitor is located at each solenoid.

The intent is that this cryomodule will have all external connections to the cryogenic, RF, and instrumentation systems made at removable junctions at the cryomodule itself. The only connection to the beamline is the beam pipe itself which will be terminated by "particle free" valves at both ends. Minimizing mean time between failure and repair and in-situ repair of some internal systems are important design considerations in the cryomodule design. Figure 1 shows the linac layout 
including the location of the SSR1 cryomodule. Table 1 lists pertinent operational requirements and parameters of the cryomodule assembly.

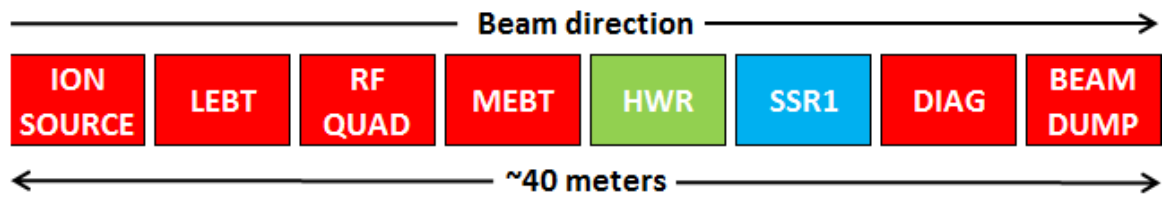

Figure 1. PXIE Layout

\begin{tabular}{|c|c|}
\hline \multicolumn{2}{|c|}{ Table 1. SSR1 Functional Requirements Specifications (subject to change) } \\
\hline \multicolumn{2}{|c|}{ General } \\
\hline Physical beam aperture, $\mathrm{mm}$ & 30 \\
\hline Overall length (flange-to-flange), $\mathrm{m}$ & $\leq 5.4$ \\
\hline Overall width, $\mathrm{m}$ & $\leq 1.6$ \\
\hline Beamline height from the floor, $\mathrm{m}$ & 1.3 \\
\hline Cryomodule height (from floor), $\mathrm{m}$ & $\leq 2.00$ \\
\hline Ceiling height in the tunnel, $\mathrm{m}$ & 3.20 \\
\hline Maximum allowed heat load to $70 \mathrm{~K}, \mathrm{~W}$ & 250 \\
\hline Maximum allowed heat load to $5 \mathrm{~K}, \mathrm{~W}$ & 80 \\
\hline Maximum allowed heat load to $2 \mathrm{~K}, \mathrm{~W}$ & 50 \\
\hline Maximum number of lifetime thermal cycles & 50 \\
\hline Intermediate thermal shield temperature, $\mathrm{K}$ & $45-80$ \\
\hline Thermal intercept temperatures, $\mathrm{K}$ & 5 and $45-80$ \\
\hline Cryo-system pressure stability at $2 \mathrm{~K}$ (RMS), mbar & $\sim 0.1$ \\
\hline Environmental contribution to internal field & $15 \mathrm{mG}$ \\
\hline Transverse cavity alignment error, mm RMS & $<1$ \\
\hline Angular cavity alignment error, mrad RMS & $\leq 10$ \\
\hline Transverse solenoid alignment error, mm RMS & $<0.5$ \\
\hline Angular solenoid alignment error, mrad RMS & $<1$ \\
\hline \multicolumn{2}{|l|}{ Cavities } \\
\hline Number, total & 8 \\
\hline Frequency, $\mathrm{MHz}$ & 325 \\
\hline$\beta$ geometric & 0.22 \\
\hline Operating temperature, $\mathrm{K}$ & 2 \\
\hline Operating mode & $\mathrm{CW}$ \\
\hline Operating energy gain at $\beta=0.22, \mathrm{MV} /$ cavity & 2 \\
\hline Coupler type - standard coaxial with impedance, $\Omega$ & 105 \\
\hline Coupler power rating, $\mathrm{KW}$ & $>20$ \\
\hline \multicolumn{2}{|l|}{ Solenoids } \\
\hline Number, total & 4 \\
\hline Operating temperature, $\mathrm{K}$ & 2 \\
\hline
\end{tabular}




\begin{tabular}{|c|c|}
\hline Current at maximum strength, $\mathrm{A}$ & $\leq 100$ \\
\hline $\int B^{2} d L, T^{2} m$ & 4.0 \\
\hline Each solenoid has independent powering & \\
\hline \multicolumn{2}{|l|}{$\begin{array}{r}\text { Correctors } \\
\end{array}$} \\
\hline Number, total & 8 \\
\hline Number, per solenoid package & 2 \\
\hline Current, A & $\leq 50$ \\
\hline Strength, T-m & 0.0025 \\
\hline \multicolumn{2}{|l|}{ Beam Position Monitors } \\
\hline Number, total & 4 \\
\hline Number of plates & 4 \\
\hline Electrical center accuracy compared to geometric center, $\mathrm{mm}$ & $\leq \pm 0.5$ \\
\hline
\end{tabular}

\section{CRYOMODULE DESIGN}

Eventually, Project $\mathrm{X}$ will require several different cryomodule designs for cavities operating at 162.5, 325, 650, and $1300 \mathrm{MHz}$. The SSR1 for PXIE is the first of these being developed at Fermilab. Some details of individual cryomodule components are described in the following sections.

\section{CRYOGENIC SYSTEMS AND VACUUM INTERFACES}

There are two ways that cryogenic and vacuum systems are distributed to individual modules in superconducting magnets or cavity strings. The first, sometimes called coarse segmentation, refers to systems in which the cryogenic circuits and insulating vacuum inside individual cryostats are more or less continuous for long lengths, at least over the length of several cryomodules. Most accelerator magnet systems are configured this way as are the cryomodules envisioned for the ILC and the XFEL at DESY. The second, referred to as fine segmentation, refers to systems in which the insulating vacuum and the cryogenic circuits are confined to an individual cryomodule, the only connection between modules being the at beam tube. The cryomodules for the SNS are one example of fine segmentation. Fine segmentation is the configuration choice for Project X and PXIE cryomodules. Each individual vacuum vessel will be closed at both ends and the cryogenic circuits will be fed through bayonet connections at each cryomodule. Each cryomodule will have its own connection to the insulating vacuum pumping system. Also, each cryomodule will have its own $2 \mathrm{~K}$ heat exchanger and pressure relief line exiting near the middle of the module. This configuration provides flexibility in terms of cryomodule replacement, and cooldown and warm-up times at the expense of requiring more individual cryogenic connections, cold-to-warm transitions at each end of each cryomodule, and extra space at each interconnect to close the beam tube.

\section{VACUUM VESSEL}


The vacuum vessel serves to house all the cryomodule components in their asinstalled positions, to provide a secure anchor to the tunnel floor, to insulate all cryogenic components in order to minimize heat load to $80 \mathrm{~K}, 4.5 \mathrm{~K}$, and $2 \mathrm{~K}$, as well as maintain the insulating vacuum. It is $1.219 \mathrm{~m}$ (48 inches) in diameter and manufactured from carbon steel and is shown as the outermost shell in figure 8 .

\section{MAGNETIC SHIELD}

Just inside the vacuum vessel, virtually in contact with the inner wall, is a magnetic shield to shield the cavities from the earth's magnetic field. Preliminary tests show that a $1.5 \mathrm{~mm}$-thick mu-metal shield at room temperature reduces the residual field inside the cryostat to less than $10 \mu \mathrm{T}$. It is likely that separate magnetic shields will be installed around individual magnetic elements to further reduce the potential for trapped fields in the superconducting cavity structures.

\section{THERMAL SHIELD AND MULTI-LAYER INSULATION}

Each cryomodule will have a single thermal shield cooled with helium gas, nominally at 45-80 K. It is currently envisioned to be made from 6000-series aluminum with cooling channels on both sides. Two 15-layer blankets of multi-layer insulation, between the vacuum vessel and thermal shield will reduce the radiation heat load from the room temperature vacuum vessel to approximately $1.5 \mathrm{~W} / \mathrm{m} 2$. A $5 \mathrm{~K}$ circuit will be available to intercept heat on the input couplers and current leads, but there is no plan to install a full $5 \mathrm{~K}$ thermal shield.

\section{SUPPORT SYSTEM}

All of the cavities and solenoids will be mounted on individual support posts which are in turn mounted to a full-length strongback located between the vacuum vessel and thermal shield. This enables the entire cavity string to be assembled and aligned as a unit then inserted into the vacuum vessel during final assembly. The strongback is aluminum to provide a uniform temperature base. Maintaining the strongback at room temperature helps minimize axial movement of the cold elements during cooldown, reducing displacement of couplers, current leads, and many of the internal piping components. 


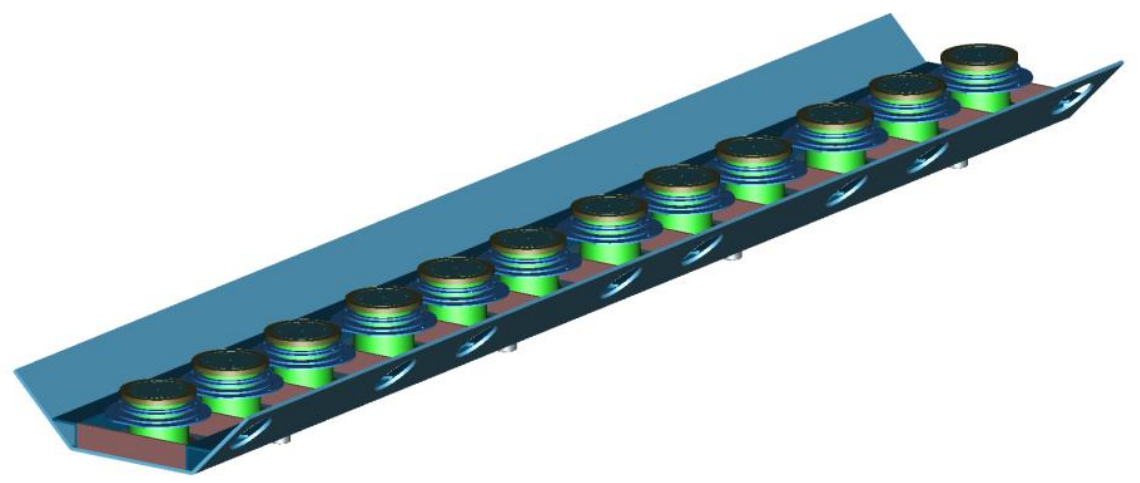

Figure 2. Strongback with Supports

The support posts are similar to supports utilized in SSC collider dipole magnets and ILC and XFEL 1.3 GHz cavity cryomodules. The main structural element is a glass and epoxy composite tube. The tube ends and any intermediate thermal intercepts are all assembled using conventional shrink-fit assembly techniques in which the composite tube is sandwiched between an outer metal ring and inner metal disk. The strongback and support posts are shown in figure 2. All of the cavities and magnetic elements are mounted to the support posts using adjustable positioning mechanisms. [1]

\section{CAVITY AND TUNER}

The cryomodule contains eight single spoke, $\beta=0.22,325 \mathrm{MHz}$ cavities operating in $\mathrm{CW}$ mode at $2 \mathrm{~K}$ in stainless steel helium vessels. Each has an integral coarse and fine tuner that operates through a lever system and pushes on the cavity end wall. For ease of maintenance, tuner access covers are incorporated into the helium vessel design. The cavity and tuner system is shown in figure 3 and described more completely in [2]. 


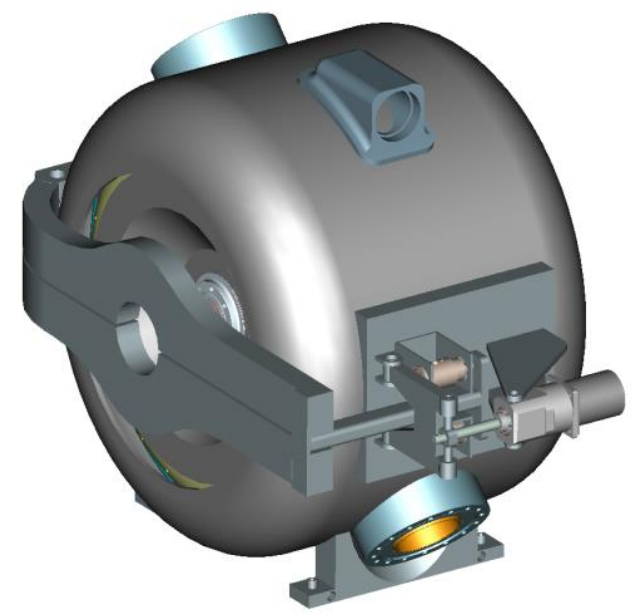

Figure 3. Spoke Cavity, Helium Vessel, and Tuner

\section{INPUT COUPLER}

The input coupler is a $105-\mathrm{ohm}$ coaxial design that supplies approximately $2 \mathrm{~kW}$ $\mathrm{CW}$ to each cavity in PXIE and ultimately up to $18 \mathrm{~kW} \mathrm{CW}$ in Project X. The coupler contains a single warm ceramic window that provides separation of the warm and cold coupler sections. During cryomodule fabrication, the cold section can be installed on the cavity in the cleanroom prior to assembly of the string. The warm section can then be installed from outside the vacuum vessel during final assembly. The inner conductor is solid copper with copper bellows to accommodate motion due to misalignment and thermal contraction. The cold end of the outer conductor is $316 \mathrm{~L}-$ stainless steel. The warm end is copper with copper bellows. Heat load estimates don't suggest a significant penalty for not copper plating the outer conductor. A forced-air cooling tube is inserted into the inner conductor after assembly that supplies air to cool the coupler tip. Figure 4 shows the current coupler design. The input coupler design is described more thoroughly in [3]. 


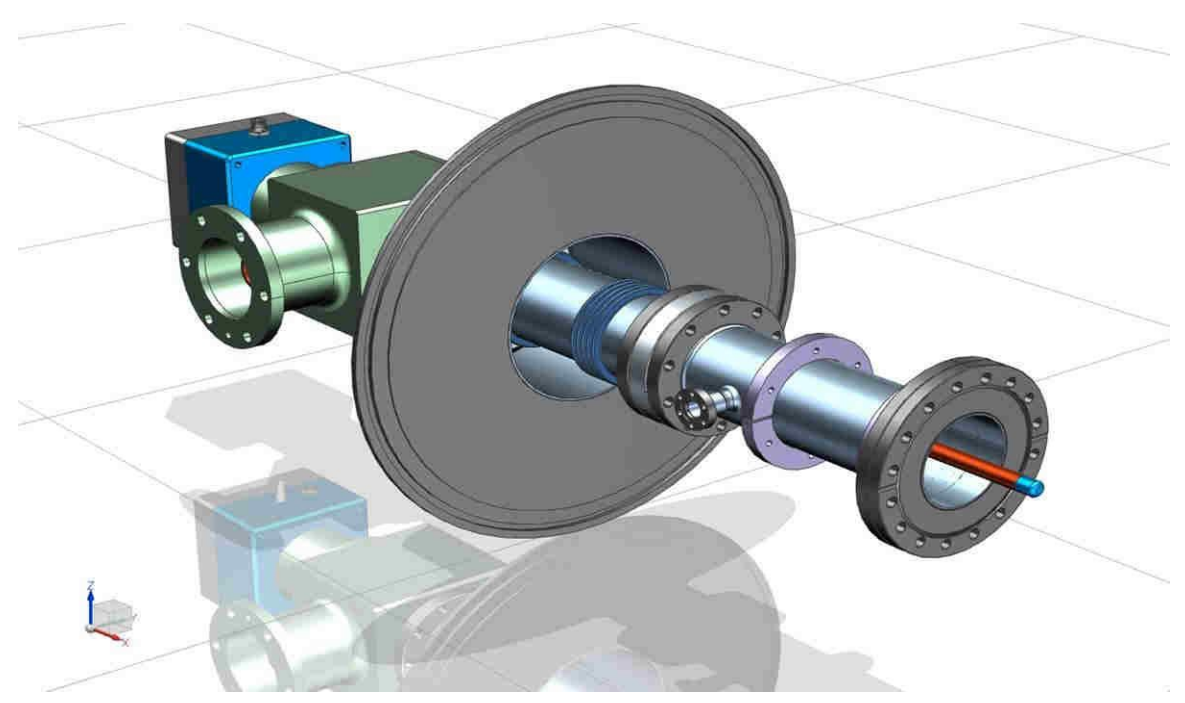

Figure 4. Input Coupler

\section{CURRENT LEADS}

Each focusing element package contains up to three magnet coils, the main solenoid, operating nominally at $100 \mathrm{~A}$ and two steering correctors each operating nominally at $50 \mathrm{~A}$. A conduction cooled current lead design modeled after similar leads installed in the LHC at CERN is being developed for use in the SSR1 cryomodule [4]. Figure 5 illustrates the design for the lead assembly. Thermal intercepts at $45-80 \mathrm{~K}$ and at $5 \mathrm{~K}$ help reduce the heat load to $2 \mathrm{~K}$, nonetheless, these current leads represent a significant source of heat at the low temperature end. There will be one lead assembly for each magnetic element.

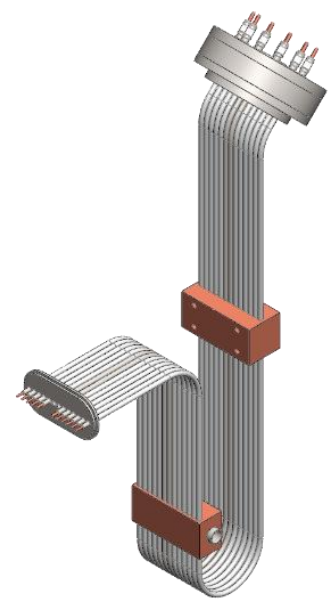


Figure 5. Conduction Cooled Current Lead Assembly

\section{SOLENOID AND BEAM POSITION MONITOR}

The four magnet packages in the cryomodule each contain a focusing solenoid and two dipole correctors all operating in a helium bath at $2 \mathrm{~K}$. The Project $\mathrm{X}$ lattice, especially the low-beta section, provides little room along the beamline for beam diagnostics either inside individual cryomodules or between adjacent modules. In order to conserve axial space along the beamline a button-type beam position monitor (BPM) was chosen for installation in the SSR cryomodules. A total of four will be installed in the cryomodule and tested in PXIE, one at each magnetic element. These devices are compact and lend themselves well to incorporation right into the solenoid magnet package as shown below in figure 6 . The bellows in either end of the beam tube allow independent adjustment of each magnet.

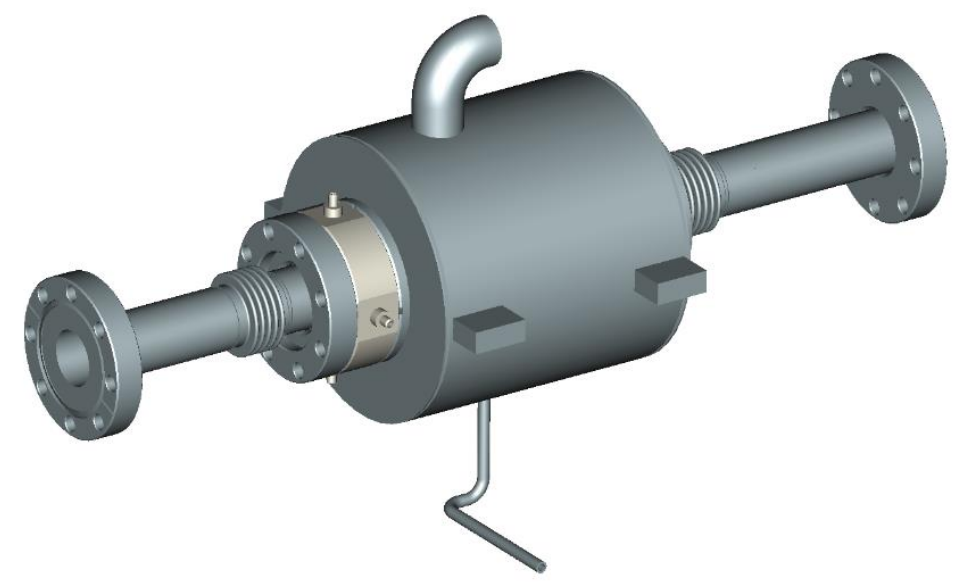

Figure 6: Solenoid and BPM assembly

\section{FINAL ASSEMBLY}

The final assembly of the SSR 1 cryomodule for PXIE is shown in figures 7 and 8. Figure 7 shows the cavity string consisting of the cavities, solenoids, beam position monitors, and internal piping mounted on support posts which are in turn mounted to the strongback. Figure 8 shows the entire cryomodule assembly. 


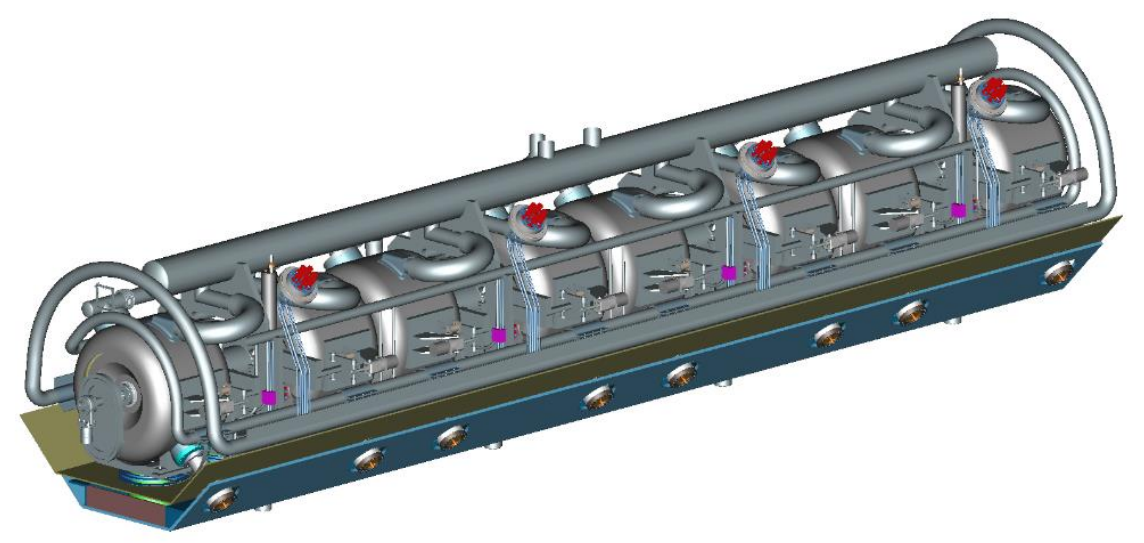

Figure 7. Cavity String Assembly

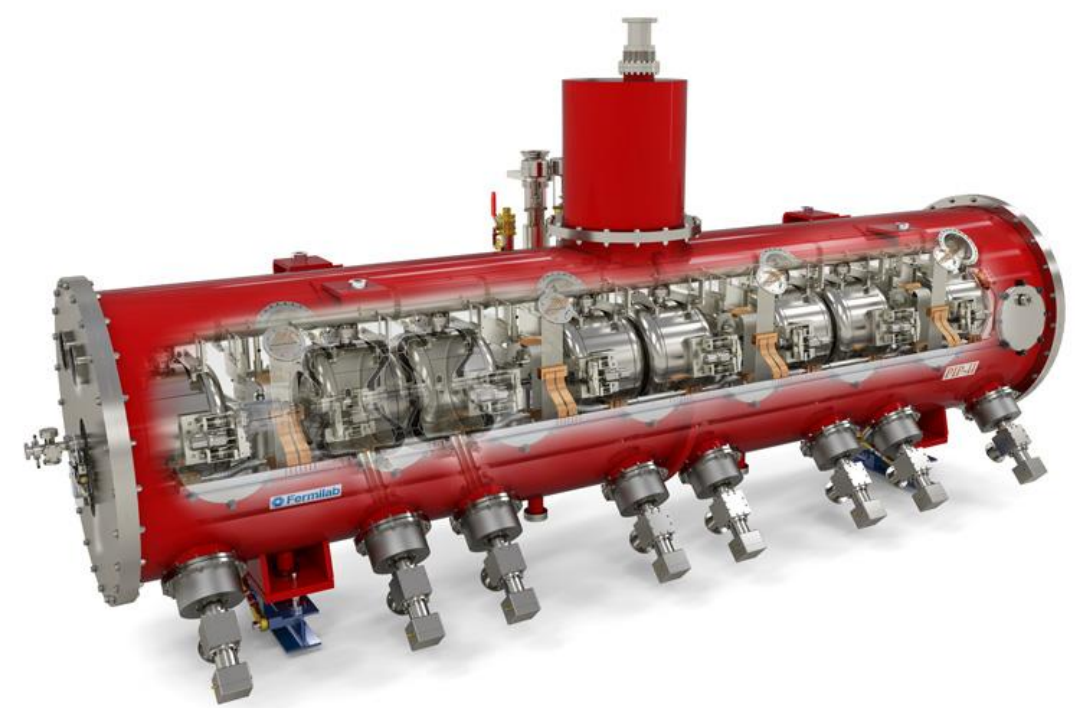

Figure 8. Cryomodule Assembly

\section{STATUS AND PLANS}

Fermilab has the PXIE SSR1 vacuum vessel, cavities, strongback, support posts, and many other cryomodule components in-house. Work is in the process to build up production facilities, develop assembly procedures for the cavity string, and to tooling for insertion of the cavity string into the cryomodule. Installation of the cryomodule in the PXIE facility is expected in 2017. 


\section{REFERENCES}

1. Nicol, T.H., Niemann, R.C., and Gonczy, J.D., "Design and Analysis of the SSC Dipole Magnet Suspension System", Supercollider 1, pp. 637649, Plenum Press, New York, 1989.

2. Ristori, L., et al, "Design of Single Spoke Resonators for PXIE", presented at IPAC 2012, paper ID: 2689-WEPPC057.

3. Kazakov, S., et al, "Main Couplers Design for Project X", presented at IPAC 2012, paper ID: 2523-WEPPC050.

4. Ballarino, A., "Conduction-Cooled 60 A Resistive Current Leads for LHC Dipole Correctors", LHC Project Report 691, 2004.

5. Nicol, T.H., Lanfranco, G., Ristori, L., "High Intensity Neutrino Source Superconducting Spoke Resonator and Test Cryostat Design and Status", IEEE Transactions on Applied Superconductivity, Volume 19, Number 3, pp. 1432-1435, 2009. 